\title{
An Interaction between the Human Cholesteryl Ester Transfer Protein (CETP) and Apolipoprotein A-I Genes in Transgenic Mice Results in a Profound CETP-mediated Depression of High Density Lipoprotein Cholesterol Levels
}

\author{
Tony Hayek, Tova Chajek-Shaul, Annemarie Walsh, Luis B. Agellon, * Philippe Moulin, * Alan R. Tall, * and Jan L. Breslow \\ Laboratory of Biochemical Genetics and Metabolism, The Rockefeller University, New York 10021; and *Division of Molecular \\ Medicine, Department of Medicine, Columbia University, New York 10032
}

\begin{abstract}
We have previously described two transgenic mouse lines, one heterozygous for the human apo A-I gene and the other heterozygous for a human cholesteryl ester transfer protein (CETP) minigene driven by the mouse metallothionein-I gene promoter. In the current study, these two lines were crossed producing control, HuCETPTg, HuAITg, and HuAICETPTg mice to study the influence of CETP on HDL cholesterol levels, particle size distribution, and metabolism in animals with mouse and human-like HDL. In the HuCETPTg and HuAICETPTg animals, zinc induction approximately doubled plasma CETP activity, with no activity in plasma from the control and HuAITg animals. The only significant effect of CETP on lipoprotein subfraction cholesterol concentrations was for HDL-C. Compared to control animals, HuCETPTg animals had lower HDL$\mathrm{C}, \mathbf{2 0 \%}$ before and $35 \%$ after $\mathrm{Zn}$ induction, and compared to HuAITg animals, HuAICETPTg animals had lower HDL-C, $35 \%$ before and $66 \%$ after $\mathrm{Zn}$ induction. Control and $\mathrm{Hu}-$ CETPTg HDL consist primarily of a single size population with a mean diameter of $10.00 \pm 0.10 \mathrm{~nm}$ and $9.71 \pm 0.05 \mathrm{~nm}$, respectively. HuAITg HDL consists primarily of three distinct HDL size subpopulations with peak diameters of $10.35 \pm 0.08$ $\mathrm{nm}, 8.80 \pm 0.06 \mathrm{~nm}, 7.40 \pm 0.10 \mathrm{~nm}$, and HuAICETPTg HDL also consists primarily of three distinct HDL size subpopulations with peak diameters of $9.87 \pm 0.05 \mathrm{~nm}, 8.60 \pm 0.10 \mathrm{~nm}$, $7.30 \pm 0.15 \mathrm{~nm}$ before, and $9.71 \pm 0.08 \mathrm{~nm}, 8.50 \pm 0.11 \mathrm{~nm}$, $7.27 \pm 0.15 \mathrm{~nm}$ after zinc induction, respectively. Western blotting analysis of nondenaturing gradient gels of plasma with a monoclonal antibody to CETP indicated that in HuCETPTg and HuAICETPTg mice, 22 and $100 \%$, respectively, of the CETP was HDL associated. Turnover studies with HDL doubly labeled with ${ }^{125} \mathrm{I}$ apo $\mathrm{A}-\mathrm{I}$ and ${ }^{3} \mathrm{H}$ cholesteryl linoleate indicated that the CETP-induced fall in HDL-C was associated with increased HDL-cholesterol ester fractional catabolic rate in both the absence and presence of human apo A-I, suggesting CETP-mediated transfer of HDL-cholesterol ester to apo Bcontaining lipoproteins. In summary, these studies suggest that CETP has a much more profound effect on HDL cholesterol levels in transgenic animals expressing human apo A-I. This
\end{abstract}

Address correspondence to Tony Hayek, M.D., Laboratory of Biochemical Genetics and Metabolism, The Rockefeller University, 1230 York Avenue, New York, NY 10021. 1992.

Received for publication 22 July 1991 and in revised form 17 March

J. Clin. Invest.

(c) The American Society for Clinical Investigation, Inc.

$0021-9738 / 92 / 08 / 0505 / 06 \$ 2.00$

Volume 90, August 1992, 505-510 may be due to an enhanced interaction of CETP with human compared to mouse apo A-I or to the HDL particles they produce. (J. Clin. Invest. 1992. 90:505-510.) Key words: high density lipoprotein • turnover • particle size distribution • metabolism • cholesteryl ester transfer protein

\section{Introduction}

The risk of coronary heart disease in humans is strongly inversely correlated with the plasma concentration of HDL cholesterol (1-3). Therefore, it is important to understand the factors that influence HDL cholesterol levels. It is postulated that HDL mediates its antiatherogenic effect by transporting cholesterol from peripheral tissues back to the liver for excretion $(4,5)$. Nascent HDL, consisting of the principal HDL apolipoprotein apo A-I and phospholipid, attracts excess free cholesterol from tissues. This is esterified by the enzyme lecithin (6). There is evidence that the liver can remove HDL cholesteryl ester from the circulation by three mechanisms: whole particle uptake (7), selective HDL cholesteryl ester removal $(8,9)$, and transfer of HDL cholesteryl ester by the cholesteryl ester transfer protein (CETP) ${ }^{1}$ to apo B-containing lipoprotein particles which are then removed by liver LDL receptors $(10,11)$. The latter pathway is thought to be very important in humans.

We have been using transgenic technology to study HDL metabolism and the role of CETP in the regulation of HDL cholesterol levels (12-15). Using human apo A-I gene constructions, we have produced transgenic mouse lines that have an increased plasma apo A-I concentration, with $90 \%$ of the apo A-I being human and mouse apo A-I greatly reduced. These transgenic lines have increased HDL cholesterol levels. The HDL consists of several distinct size subpopulations resembling human $\mathrm{HDL}_{2}$ and $\mathrm{HDL}_{3}$, whereas control mouse HDL consists of single size population. Recently, Rubin et al. (14) have made transgenic mice by similar techniques and have reported the same observation. We have also shown that these mice have lost the HDL cholesteryl ester selective uptake pathway (13).

In other experiments, we have used a human CETP minigene construction, under the control of the mouse metallothionein-I gene promoter, to produce a transgenic mouse line with plasma levels of CETP activity comparable to those in humans (15). These levels can be increased approximately twofold by zinc induction. Normal mice do not show plasma CETP activity. To our surprise, the HuCETPTg mice had

1. Abbreviations used in this paper: CE, cholesterol ester; CETP, cholesteryl ester transfer protein; FC, free cholesterol; FCR, fractional catabolic rate. 
HDL cholesterol levels comparable to control animals. A significant reduction of HDL cholesterol concentrations was observed only after zinc induction. This suggested that mouse HDL might be a poor substrate for CETP compared to human HDL. To test this hypothesis, we crossed HuAITg with $\mathrm{Hu}-$ CETPTg mice. On the background of the human apo A-I gene, CETP expression caused pronounced lowering of HDL cholesterol levels, $38 \%$ before and $66 \%$ after $\mathrm{Zn}$ induction. These results suggest that human-like HDL is a better substrate than mouse HDL for CETP and provide direct proof that CETP can cause a profound lowering of HDL cholesterol concentrations.

\section{Methods}

Animals. We previously described five lines of human apo A-I transgenic (HuAITg) mice (12). Heterozygote animals from a line with the highest levels of human apo A-I expression, $\operatorname{Tg} 179$, were used for mating with CETP heterozygote transgenic mice, to produce four groups of animals: $(a)$ Nontransgenic mice (control); $(b)$ human CETP transgenic mice (HuCETPTg); $(c)$ human apo A-I transgenic mice (HuAITg); (d) human apo A-I/CETP transgenic mice (HuAICETPTg).

Transgenic animals were identified by Southern blotting analysis of tail tip DNA to identify the human apo A-I (12) or the human CETP gene (15) or both. Animals were placed in metabolic cages and given free access to food and water. Mice 12-16-wk old were given a regular mouse chow diet (Purina Chow 5001; Ralston Purina Co., St. Louis, $\mathrm{MO}$ ), and distilled water for $7 \mathrm{~d}$, and then a chow diet and water supplemented with $25 \mathrm{mM} \mathrm{ZnSO}_{4}$ for $7 \mathrm{~d}$ to raise the level of plasma CETP. At the end of each 7-d period, the mice were fasted overnight and blood was collected the following morning from the retroorbital plexus under methoxyflurane anesthesia.

Plasma lipid and lipoprotein analysis. Total cholesterol and free cholesterol (FC) levels were determined enzymatically using reagents (Boehringer Mannheim Biochemicals, Indianapolis, IN) (12). For small volumes of mouse plasma, HDL was separated from apo B-containing lipoproteins by dextran sulfate precipitation as described previously $(12,13)$. Other lipoproteins were isolated by ultracentrifugation in an airfuge (Beckman Instruments, Palo Alto, CA ) as described previously (12). For large scale separation, lipoproteins (VLDL, $d<1.006$ $\mathrm{g} / \mathrm{ml} ; \mathrm{LDL}, d=1.006-1.063 \mathrm{~g} / \mathrm{ml}$; and HDL, $d=1.063-1.21 \mathrm{~g} / \mathrm{ml}$ ) from $1 \mathrm{ml}$ of pooled transgenic or nontransgenic mouse plasma were isolated by sequential density ultracentrifugation.

To estimate lipoprotein size, plasma lipoproteins were stained with Sudan black before electrophoresis of plasma on native 4-30\% polyacrylamide gradient gels (Pharmacia LKB, Piscataway, NJ) $(16,17)$. To determine CETP localization in mouse plasma, after gradient gel electrophoresis the lipoproteins were blotted onto nitrocellulose. The blot was then probed with ${ }^{125}$ I-TP2, an mAb that recognizes an epitope near the carboxy terminus of human CETP (18), and scanned using a laser densitometer (Molecular Dynamics, Sunnyvale, CA).

Apo A-I quantitation. Human apo A-I was quantitated by a sandwich ELISA using a polyclonal goat anti-human apo A-I antibody that had $<0.01 \%$ cross-reactivity with mouse apo A-I (12). Mouse apo A-I was quantitated by rocket electroimmunoassay (13) using a polyclonal anti-mouse apo A-I antibody prepared in cynomolgus monkeys, and generously supplied by Dr. George Melchior of the Upjohn Company, Kalamazoo, MI. This antibody had $<0.01 \%$ cross-reactivity with human apo A-I.

Plasma CETP concentration and activity. Plasma CETP concentration was measured by solid phase radioimmunoassay as described previously (19). Measurement of CETP activity in mouse plasma was done as described by Agellon et al. (15).

Preparation of HDL labeled in the apo A-I and cholesteryl ester moieties. Human apo A-I was purified and radiolabeled with ${ }^{125} \mathrm{I}$ by the Bilheimer modification of the McFarlane method as previously de- scribed $(20,21)$. The specific activity of ${ }^{125}$ I-labeled apo A-I was $\sim 200$ $\mathrm{cpm} / \mathrm{ng} .10 \mu \mathrm{Ci}$ of $\left[{ }^{3} \mathrm{H}\right]$ cholesteryl linoleate was placed in a glass tube and the solvent was dried under $\mathrm{N}_{2} .1 \mathrm{ml}$ of $0.15 \mathrm{M}$ of $\mathrm{NaCl}$ containing $125 \mu \mathrm{g}$ triacylglycerol (Intralipid; Kabi Vitrum Inc., Stockholm, Sweden) was added. The triacyglycerol was labeled with $\left[{ }^{3} \mathrm{H}\right]$ cholesteryl linoleate by sonication 12 times ( $10 \mathrm{~s}$ each) using a sonicator (Branson Ultrasonics Corp., Danbury, CT) at $50 \%$ output in ice-cooled water. To label HDL with $\left[{ }^{3} \mathrm{H}\right]$ cholesteryl linoleate, $1 \mathrm{ml}$ of plasma from control or HuAITg mice was incubated with labeled Intralipid ( $125 \mu \mathrm{g}$ triacylglycerol) and $1 \mathrm{ml}$ of $\mathrm{d}>1.25 \mathrm{~g} / \mathrm{ml}$ rabbit plasma (a source of CETP) for $16 \mathrm{~h}$ at $37^{\circ} \mathrm{C}$ as described previously (22). The labeled HDL fraction was isolated by sequential ultracentrifugation between $\mathrm{d} 1.063$ and $1.21 \mathrm{~g} / \mathrm{ml}$, dialyzed against five changes of 1 liter of $0.9 \% \mathrm{NaCl}, 1$ mM EDTA over $20 \mathrm{~h}$, and used immediately. 2-4 $\mu \mathrm{g}$ of [ $\left.{ }^{125} \mathrm{I}\right]$ apo A-I was mixed with 100,000-200,000 dpm (10-20 $\mu \mathrm{g}$ HDL-C) of the [ $\left.{ }^{3} \mathrm{H}\right]-$ cholesteryl linoleate-labeled HDL before injection. This procedure was associated with a $>90 \%$ recovery of $\left[{ }^{3} \mathrm{H}\right]$ cholesteryl linoleate in the plasma $10 \mathrm{~min}$ after its injection (22).

In vivo turnover studies. Mice were injected intravenously (femoral vein) with either control or HuAITg mouse HDL doubly labeled with $2-4 \mu \mathrm{g}$ of $\left[{ }^{125} \mathrm{I}\right]$ human apo A-I and $100,000-200,000 \mathrm{dpm}$ of $\left[{ }^{3} \mathrm{H}\right]-$ cholesteryl linoleate. In previous work, we had shown by ultracentrifugation that both labels remained with the HDL fraction (d 1.063-1.21 $\mathrm{g} / \mathrm{ml}$ ) during the time course of the experiment (13). We also showed that the apo A-I tracer comigrates with HDL by gradient gel electrophoresis (13). The injected HDL mass was $<5 \%$ of the mouse HDL pool. Blood $(50 \mu \mathrm{l})$ was taken from the retroorbital plexus under anesthesia with methoxyflurane at $10 \mathrm{~min}, 90 \mathrm{~min}, 3 \mathrm{~h}, 8 \mathrm{~h}$, and $28 \mathrm{~h}$ for determination of radioactivity (13). The fractional catabolic rates for apo A-I and HDL cholesteryl linoleate were calculated from the decay curves of $\left[{ }^{125} \mathrm{I}\right]$ apo A-I and $\left[{ }^{3} \mathrm{H}\right]$ cholesteryl linoleate radioactivity in whole plasma and in the HDL fraction after dextran sulfate precipitation of apo B-containing lipoproteins, assuming a two-pool model by the Matthews method (23).

\section{Results}

To better understand the effect of CETP on HDL metabolism, four groups of animals were studied: control, HuCETPTg, HuAITg, and HuAICETPTg. For each group, total plasma and lipoprotein subfraction cholesterol concentrations and plasma apo A-I levels were determined on a chow diet before and after $\mathrm{Zn}$ induction (Table I). In the HuCETPTg and HuAICETPTg animals, $\mathrm{Zn}$ induction approximately doubled the CETP activity in plasma. Plasma from the other animals had no measurable CETP activity. The only significant effect of CETP on the lipoprotein subfraction cholesterol concentration was for HDL-C. Control animals on chow had HDL-C of $49 \pm 3 \mathrm{mg} / \mathrm{dl}$ whereas HuCETPTg animals had HDL-C of $39 \pm 3 \mathrm{mg} / \mathrm{dl}$. This reduction of $20 \%$ was just significant $(P<0.05)$. $\mathrm{Zn}$ treatment of the HuCETPTg animals caused a further HDL-C reduction to $32 \mathrm{mg} / \mathrm{dl}$, or a total of $35 \%(P<0.005)$. HuAITg animals on chow had HDL-C of $109 \pm 9 \mathrm{mg} / \mathrm{dl}$, whereas HuAICETPTg animals had HDL-C of $68 \pm 5 \mathrm{mg} / \mathrm{dl}$. This reduction of $38 \%$ was highly significant $(P<0.005)$. Even more impressive, $\mathrm{Zn}$ treatment of the HuAICETPTg animals caused a further HDLC reduction to $37 \pm 4 \mathrm{mg} / \mathrm{dl}$, or a total of $66 \%(P<0.001)$. Since the plasma CETP activity was roughly comparable in HuCETPTg and HuAICETPTg mice, these experiments suggest that CETP has a much more profound HDL-C lowering effect in transgenic animals expressing human apo A-I.

FC and cholesterol ester (CE) composition analysis of transgenic and nontransgenic mouse pooled plasma revealed a significant decrease in FC/CE ratio in HuCETPTg mice (50\% 
Table I. Lipoprotein Profile, Apo A-I Levels, and CETP Activity in Control and Human CETP, A-I, and A-I CETP Transgenic Mice

\begin{tabular}{|c|c|c|c|c|c|c|c|c|}
\hline Mice & $\mathrm{ZnSO}_{4}$ & Total cholesterol & HDL-C & LDL-C & VLDL-C & Human A-I & Mouse A-I & CETP activity \\
\hline & & \multicolumn{6}{|c|}{$m g / d l$} & $\mathrm{cpm} / \mu \mathrm{l}$ \\
\hline Control & - & $64 \pm 4$ & $49 \pm 3$ & $7 \pm 1$ & $8 \pm 2$ & - & $180 \pm 11$ & - \\
\hline$(n=13)$ & + & $72 \pm 3$ & $55 \pm 3$ & $7 \pm 1$ & $8 \pm 2$ & - & $174 \pm 14$ & - \\
\hline HuCETPTg & - & $50 \pm 3^{*}$ & $39 \pm 3^{*}$ & $5 \pm 1$ & $6 \pm 1$ & - & $177 \pm 18$ & $204 \pm 19$ \\
\hline$(n=9)$ & + & $41 \pm 3^{\ddagger}$ & $32 \pm 2^{\ddagger}$ & $4 \pm 1$ & $6 \pm 1$ & - & $137 \pm 13$ & $392 \pm 32^{\S}$ \\
\hline HuAITg & - & $136 \pm 11$ & $109 \pm 9$ & $7 \pm 1$ & $19 \pm 5$ & $283 \pm 37$ & $5 \pm 4$ & - \\
\hline$(n=9)$ & + & $130 \pm 12$ & $101 \pm 10$ & $12 \pm 3$ & $17 \pm 3$ & $281 \pm 32$ & $14 \pm 4$ & - \\
\hline HuAICETPTg & - & $83 \pm 5^{\prime \prime}$ & $68 \pm 5^{\prime}$ & $8 \pm 1$ & $7 \pm 2$ & $217 \pm 24$ & $10 \pm 3$ & $260 \pm 35$ \\
\hline$(n=8)$ & + & $49 \pm 4^{* *}$ & $37 \pm 4^{* *}$ & $7 \pm 2$ & $5 \pm 1$ & $165 \pm 11^{\prime}$ & $8 \pm 3$ & $490 \pm 23^{\ddagger \ddagger}$ \\
\hline
\end{tabular}

${ }^{*} P<0.05$ when compared to control non-Zn-treated; ${ }^{\ddagger} P<0.005$ when compared to control non-Zn-treated; ${ }^{\S} P<0.01$ when compared to non-Zn-treated; " $P<0.001$ when compared to HuAITg non-Zn-treated; ' $P<0.005$ when compared to HuAITg non-Zn-treated; ${ }^{* *} P<0.0001$ when compared to HuAITg non-Zn-treated; ${ }^{\sharp} P<0.05$ when compared to non-Zn-treated.

of the value in nontransgenic controls) and in HuAICETPTg mice ( $69 \%$ of the value in HuAITg mice). Analysis of HDL, fractionated from pooled plasma of the four groups by sequential ultracentrifugation, revealed the same trend as in the plasma. Recently, Agellon et al. have demonstrated reduction of $\mathrm{FC} / \mathrm{CE}$ ratio in the plasma and all lipoprotein fractions in the HuCETPTg compared to nontransgenic controls, suggesting that lecithin:cholesterol acyltransferase activity may be stimulated in mice expressing the CETP gene (15).

The effect of CETP on plasma apo A-I levels was also studied. Control animals on chow had mouse apo A-I levels of $180 \pm 11 \mathrm{mg} / \mathrm{dl}$. HuCETPTg animals had virtually the same apo A-I levels of $177 \pm 18 \mathrm{mg} / \mathrm{dl}$ without $\mathrm{Zn}$ treatment. After $\mathrm{Zn}$ treatment, there was a trend to reduced apo A-I levels of $137 \pm 13 \mathrm{mg} / \mathrm{dl}$, a $24 \%$ reduction, but this was not significant ( $P$ $=0.062$ ). HuAITg animals on chow had human apo A-I levels of $283 \pm 37 \mathrm{mg} / \mathrm{dl}$. The HuAICETPTg animals showed a trend to reduced human apo A-I levels of $217 \pm 24 \mathrm{mg} / \mathrm{dl}$, a $23 \%$ reduction, without $\mathrm{Zn}$ treatment, but this was not significant $(P=0.18)$. After $\mathrm{Zn}$ treatment, human apo A-I levels were reduced to $165 \pm 11 \mathrm{mg} / \mathrm{dl}$, a $42 \%$ reduction, and this was significant $(P<0.005)$. Thus, as with HDL-C, CETP has a greater apo A-I lowering effect in transgenic animals expressing human apo A-I. In addition, in terms of the percent reduction, CETP appears to have a greater effect on HDL-C levels than on apo A-I levels.

Nondenaturing gradient gel electrophoresis was used to examine the effect of CETP on HDL particle size distribution (Fig. 1). Mouse plasma HDL consists primarily of a single size population with a mean diameter of $10.00 \pm 0.10 \mathrm{~nm}$. HDL in the HuCETPTg animals also consists primarily of a single size population with a smaller mean diameter of $9.71 \pm 0.05 \mathrm{~nm}$. This did not change significantly after Zn treatment. As previously reported (13), HuAITg mouse plasma HDL consists primarily of three distinct HDL subpopulations similar to those seen in human plasma. These are $\mathrm{HDL}_{2 b} 10.35 \pm 0.08 \mathrm{~nm}$, $\mathrm{HDL}_{3 \mathrm{a}} 8.80 \pm 0.06 \mathrm{~nm}$, and $\mathrm{HDL}_{3 \mathrm{c}} 7.40 \pm 0.10 \mathrm{~nm}$. The HuAICETPTg animals showed HDL particle size of $9.87 \pm 0.05 \mathrm{~nm}$, $8.60 \pm 0.10 \mathrm{~nm}$, and $7.30 \pm 0.15 \mathrm{~nm}$ before $\mathrm{Zn}$ treatment and $9.71 \pm 0.08 \mathrm{~nm}, 8.5 \pm 0.11 \mathrm{~nm}$, and $7.27 \pm 0.11 \mathrm{~nm}$ after $\mathrm{Zn}$ treatment. Thus, CETP appears to cause a profound reduction in the size of the HDL particles containing human apo A-I, especially the $\mathrm{HDL}_{2 \mathrm{~b}}$ particles.
The experiments cited thus far suggest a special interaction of CETP with the human-like HDL particles. To further explore this possibility, the native gradient gel electrophoretograms referred to above were Western blotted onto nitrocellulose and reacted with a radioiodinated monoclonal antibody to CETP (mAb TP2). After autoradiography, with plasma from the HuCETPTg mice only $\sim 22 \%$ of the radioactivity was associated with HDL, whereas with plasma from the HuAICETPTg mice $100 \%$ was HDL associated (Fig. 1). There was also a different pattern of CETP binding to HDL compared with HuCETPTg mice. This experiment is compatible with the

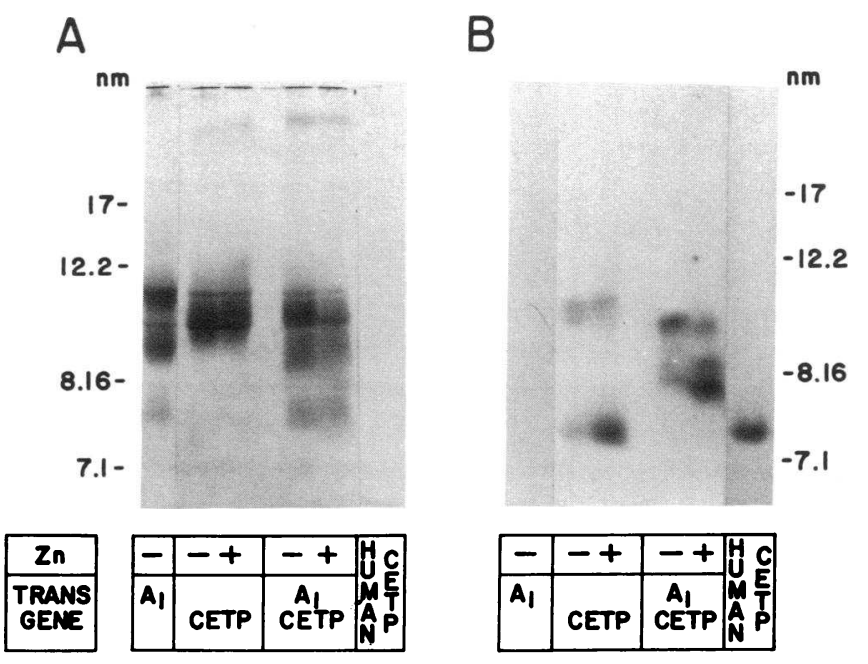

Figure 1. Association of CETP with HDL in HuCETPTg and HuAICETPTg mice. $A$ shows a native gradient gel electrophoretogram (4$30 \%$ ) of $25-\mu$ l plasma samples prestained with Sudan black. As can be seen, HuCETPTg HDL (before and after $\mathrm{Zn}$ treatment) consists primarily of a single size population, while HuAITg and HuAICETPTg HDL (before and after $\mathrm{Zn}$ treatment) consist primarily of three distinct HDL subpopulations. Molecular weight markers are indicated on the left hand side of the figure. $B$ shows an autoradiogram of the gel shown in $A$ after Western blotting with a radioiodinated antibody to CETP. The migration of free CETP in this system is shown on the right hand side of the figure. The figure shows that only a small fraction of CETP is associated with HDL in the HuCETPTg mice, whereas it is all HDL associated in the HuAICETPTg mice. 
others and provides direct evidence that CETP interacts better with human compared to mouse apo A-I containing HDL.

The effect of CETP on HDL metabolism was next examined. HDL was doubly labeled with ${ }^{125}$ I human apo A-I and $\left[{ }^{3} \mathrm{H}\right]$ cholesteryl linoleate to follow the metabolism of the HDL protein and cholesteryl ester moieties. As shown by the decay curve in Fig. $2 A$ for whole plasma and Fig. $2 C$ for the HDL fraction and Table II, in control animals, the apo A-I fractional catabolic rate (FCR) was $0.102 \pm 0.007$ pools $/ \mathrm{h}$ but the HDLCE FCR was $0.220 \pm 0.006$ pools/ $h$ indicating particulate as well as selective catabolic pathways for HDL-C. In HuAICETPTg animals, apo A-I FCR was essentially unchanged at $0.107 \pm 0.007$ pools $/ h$, whereas the HDL-CE FCR increased to $0.270 \pm 0.010$ pools $/ h$, indicating an additional catabolic pathway for HDL cholesteryl esters. This is presumably by CETP-
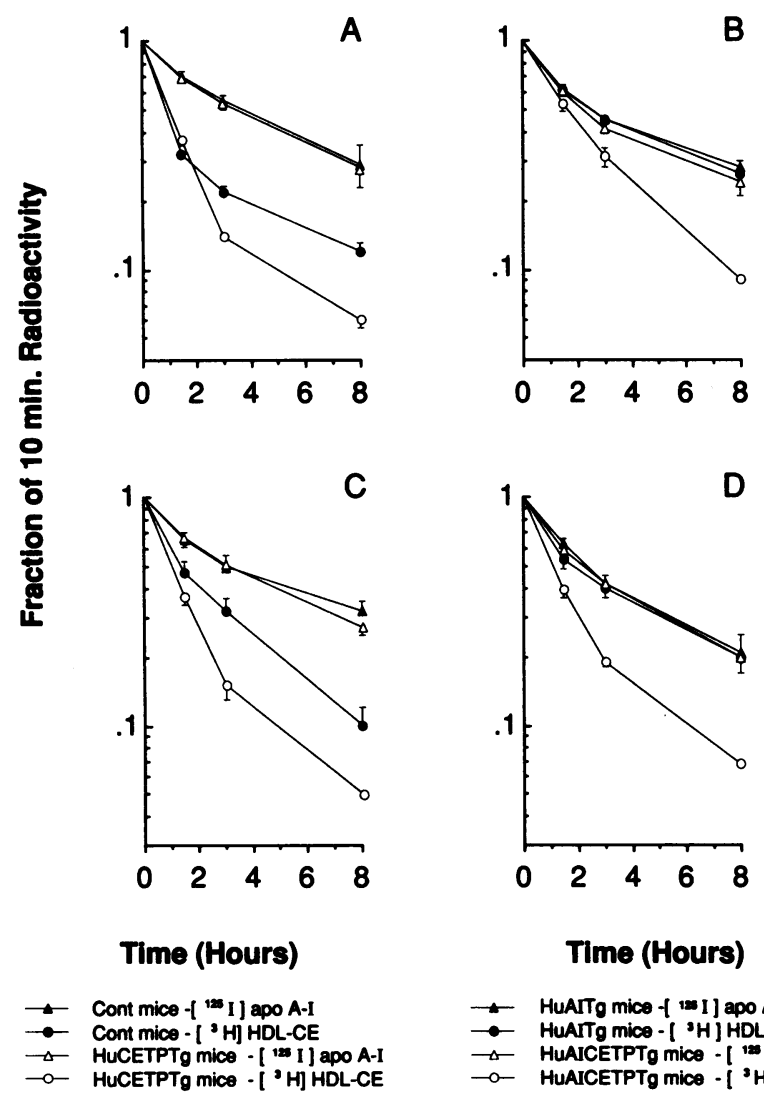

- HuAITo mico $[19 \mathrm{I}]$ apo A-I

$\rightarrow$ HuATTg mico-I 'H] HDL-CE

$\rightarrow$ HUAICETPTg mice - [ $\left.{ }^{120} \mathrm{I}\right]$ apo A-I

-O- HUAICETPTg mico - [ ' $\mathrm{H}] \mathrm{HDL}-\mathrm{CE}$

Figure 2. Radioactive decay curves for doubly labeled HDL in control and transgenic mice. Mice were injected intravenously with [ $\left.{ }^{125} \mathrm{I}\right]$ human apo A-I, $\left[{ }^{3} \mathrm{H}\right]$ cholesteryl linoleate-labeled HDL, and $50 \mu 1$ of blood was taken from the retroorbital plexus at $10 \mathrm{~min}, 90 \mathrm{~min}, 3$ $\mathrm{h}$, and $8 \mathrm{~h}$. Control and HuCETPTg mice were injected with doubly labeled control mouse HDL and radioactivity in whole plasma $(A)$ or in HDL after precipitation of apo B-containing lipoproteins $(C)$ are shown. As can be seen, the disappearance of HDL CE radioactivity is greater than for apo A-I in both control and HuCETPTg mice. Compared to controls, the HDL CE disappearance is greater in the HuCETPTg mice, but apo A-I disappearance is the same. HuAITg and HuAICETPTg mice were injected with doubly labeled HuAITg mouse HDL and radioactivity in whole plasma $(B)$ or in HDL $(D)$ are shown. As can be seen, the disappearance of HDL CE radioactivity is the same as apo A-I in HuAITg mice. Compared to these animals, HuAICETPTg mice show a large increase in HDL CE clearance and a small increase in apo A-I clearance. mediated transfer to apo B-containing particles which are then removed by liver LDL receptors. When comparing the control and HuCETPTg animals, the 23\% increase in HDL-CE FCR accounted for a little over half of the $41 \%$ decrease in HDL-C levels. As shown by the decay curve in Fig. $2 B$ for whole plasma and Fig. $2 D$ for the HDL fraction and Table II, in HuAITg animals the apo A-I FCR was $0.120 \pm 0.005$ with about the same HDL-CE FCR of $0.093 \pm 0.008$ pools $/ h$, indicating a loss of the HDL-CE selective uptake pathway in these animals, as we previously found (13). In HuAICETPTg animals, apo A-I FCR increased to $0.130 \pm 0.004(P<0.05)$, whereas HDLCE FCR increased to $0.182 \pm 0.001$ pools $/ \mathrm{h}$. This suggests that HDL cholesteryl ester removal mediated by CETP is a major pathway in these animals. When comparing the HuAITg and HuAICETPTg animals, the $96 \%$ increase in HDL-CE FCR accounted for most of the $65 \%$ decrease in HDL-C levels, whereas the $8 \%$ increase in apo A-I FCR accounted for only a small fraction of the $36 \%$ decrease in apo A-I levels. Thus CETP expression in mice lowers HDL-C levels largely by increasing HDL-CE FCR. However, CETP expression in mice lowers apo A-I levels principally by another mechanism.

\section{Discussion}

Cross-breeding two previously described transgenic mouse lines provided us the opportunity to examine the effects of CETP in vivo on human-like and mouse HDL. The greater decrease in HDL cholesterol and apo A-I concentrations in HuAICETPTg versus HuCETPTg mice suggests a specific and strong interaction between CETP and lipoproteins containing human apo A-I in vivo. We have considered two possible explanations for the nature of this interaction. It could simply be an increased amount of the HDL substrate in the HuAICETPTg versus HuCETPTg animals. This is doubtful because in our previous study (15), HuCETPTg animals were fed a high fat diet that raised HDL cholesterol to a level greater than that in the HuAICETPTg animals without increasing the effect of CETP. In addition, Barter has shown that in absolute terms CETP-mediated CE transfer in vitro is actually decreased as HDL concentration is increased (24). A second explanation, which we favor, is that HDL containing human apo A-I is a better substrate for CETP than mouse HDL. This is probably because human HDL binds CETP more effectively than mouse HDL. As we showed, Western blotting of nondenaturing gradient gels of plasma from transgenic animals with an antibody to CETP revealed $100 \%$ associated with $\mathrm{HDL}$ in the $\mathrm{HuAI}$ CETPTg mice and only $22 \%$ in the HuCETPTg mice. This is compatible with previous studies that have shown that the ability of various lipoproteins to act as substrates for CETP parallels their ability to bind CETP (25-27).

It is not certain why HDL containing human apo A-I in mouse plasma should bind CETP more effectively. The human apo A-I transgenic animals' HDL consists primarily of three distinct subpopulations, whereas nontransgenic mouse HDL consists primarily of a single size population $(13,14)$. It is possible that a particular size of HDL present in human apo A-I transgenics is optimal for CETP binding. However, in the present study the size of the largest HDL particles is quite comparable between HuAICETPTg and HuCETPTg mice, especially after $\mathrm{Zn}$ induction. Therefore, it is unlikely that the variation in CETP affinity for the different HDLs is simply due to 
Table II. HDL-CE and Apo A-I FCR in Control and Human CETP, A-I, and A-I CETP Transgenic Mice

\begin{tabular}{|c|c|c|c|c|c|}
\hline Mice & Total cholesterol & HDL-C & HDL-CE FCR & Human A-I & A-I FCR \\
\hline & \multicolumn{2}{|c|}{$m g / d l$} & pools $/ h$ & $m g / d l$ & pools $/ h$ \\
\hline Control $(n=4)$ & $76 \pm 4$ & $58 \pm 2$ & $0.220 \pm 0.006$ & - & $0.102 \pm 0.007$ \\
\hline HuCETPTg $(n=7)$ & $43 \pm 4^{*}$ & $34 \pm 3^{*}$ & $0.270 \pm 0.01^{\ddagger}$ & - & $0.107 \pm 0.007$ \\
\hline $\operatorname{HuAITg}(n=5)$ & $132 \pm 18$ & $107 \pm 14$ & $0.093 \pm 0.008$ & $266 \pm 42$ & $0.120 \pm 0.005$ \\
\hline HuAICETPTg $(n=6)$ & $51 \pm 3^{8}$ & $37 \pm 3^{6}$ & $0.182 \pm 0.001^{11}$ & $170 \pm 15^{\prime \prime}$ & $0.130 \pm 0.004^{\prime}$ \\
\hline
\end{tabular}

HDL-CE and A-I FCR were calculated from the plasma decay curve including the 28-h point. All animals received $\mathrm{ZnSO}_{4}$ for $7 \mathrm{~d}$ before the turnover study was performed.

${ }^{*} P<0.0001$ when compared to control; ${ }^{\ddagger} P<0.005$ when compared to control; ${ }^{8} P<0.0001$ when compared to HuAITg; $P<0.005$ when compared to HuAITg; ' $P<0.05$ when compared to HuAITg.

HDL particle size. Differences in affinity are probably due to HDL composition. For example, unbalanced production of apo A-I versus apo A-II could lead to an excess of Lp A-I versus Lp A-I and A-II particles in plasma. It has been shown that the former bind CETP better than the latter. Another possibility is that differences in the primary protein sequence between mouse and human apo A-I could determine the difference in CETP binding. In either case, these results indicate an activation or binding of CETP by apo A-I that was previously unsuspected. The transgenic model has unmasked this phenomenon either because of the lack of an inhibitory factor (i.e., apo A-II) or because the interaction is species specific.

The genetic factors that regulate HDL cholesterol levels and particle size distribution are of great clinical importance. Recently, homozygotes with genetic CETP deficiency, heterozygotes, and controls were shown to have CETP concentrations of $0,1.4$, and $2.3 \mu \mathrm{g} / \mathrm{ml}$, respectively, and HDL cholesterol of $164 \pm 39,66 \pm 15$, and $53 \pm 14 \mathrm{mg} / \mathrm{dl}$, respectively (27). This shows that CETP lowers HDL cholesterol levels in humans. In the human range of CETP activity, this effect can be as much as $68 \%$. In this context, our previous results with the HuCETPTg mice were disappointing because over a similar range of CETP activity HDL cholesterol lowering ranged from insignificant to approximately $30 \%$. In the current study, on the background of the human apo A-I gene, CETP was able to lower HDL cholesterol $66 \%$, a result comparable to the human genetic data (27). This suggests that CETP apo A-I gene interactions may regulate HDL cholesterol levels in humans.

In the mouse studies, HDL cholesterol lowering by CETP was in excess of that for apo A-I. This suggests that CETP reduces HDL particle size. This was in fact observed with a dramatic reduction of $\mathrm{HDL}_{2 b}$ from a particle diameter of 10.35 to $9.71 \mathrm{~nm}$ by CETP in the human apo A-I transgenics. CETP reduction of HDL particle size has been documented in humans (27). In vivo turnover studies, indicate that the HDL cholesterol lowering effect of CETP is primarily by increasing HDL-CE FCR. This is presumably by CETP-mediated transfer of HDL-CE to apo B containing lipoproteins. It is somewhat perplexing that this is not accompanied by a significant increase in plasma levels of VLDL or LDL cholesterol in the transgenic mice. In the genetic studies in humans previously cited, there was a gene dosage effect of CETP activity on plasma levels of LDL cholesterol (27). It is possible that low levels or rapid turnover of apo B-containing lipoproteins in the mouse preclude our ability to see a CETP-mediated increase in VLDL or LDL cholesterol levels. Perhaps other genes that regu- late the metabolism of these lipoproteins are necessary to see an effect of CETP.

In summary, CETP appears to have a specific and enhanced interaction with HDL particles containing human apo A-I. This suggests that effects of CETP on lipoprotein and tissue lipid metabolism may best be studied in the doubly transgenic HuAICETPTg mice described in this report.

\section{Acknowledgments}

This research was supported by National Institutes of Health grants HL-43165 and HL-21006 (A. R. Tall), and HL-32435 and HL-33714 (J. L. Breslow).

\section{References}

1. Gordon, D. J., and B. M. Rifkind. 1989. High density lipoprotein-the clinical implications of recent studies. N. Engl. J. Med. 321:1311-1316.

2. Castelli, W. P., R. J. Garrison, P. W. F. Wilson, R. D. Abbott, S. Kalousdian, and W. B. Kannel. 1986. Incidence of coronary heart disease and lipoprotein cholesterol levels. JAMA (J. Am. Med. Assoc.) 256:2835-2838.

3. Miller, N. E. 1987. Associations of high-density lipoprotein subclasses and apolipoproteins with ischemic heart disease and coronary atherosclerosis. $\mathrm{Am}$. Heart. J. 113:589-597.

4. Tall, A. R. 1990. Plasma high density lipoproteins, metabolism, and relationship to atherogenesis. J. Clin. Invest. 86:379-384.

5. Reichl, D., and N. E. Miller. 1989. Pathophysiology of reverse cholesterol transport, insights from inherited disorders of lipoprotein metabolism. Arteriosclerosis. 9:785-797.

6. Eisenberg, S. 1984. High density lipoprotein metabolism. J. Lipid Res. 25:1017-1058.

7. Eisenberg, S., Y. Oschry, and J. Zimmerman. 1984. Intravascular metabolism of the cholesteryl ester moiety of rat plasma lipoproteins. J. Lipid Res. 25:121-128.

8. Glass, C. K., R. C. Pittman, D. B. Weinstein, and D. Steinberg. 1983. Dissociation of tissue uptake of cholesterol ester from that of apoprotein A-I of rat plasma high density lipoprotein: selective delivery of cholesterol to liver, adrenal, and gonad. Proc. Natl. Acad. Sci. USA. 80:5435-5439.

9. Glass, C. K., R. C. Pittman, M. Civen, and D. Steinberg. 1985. Uptake of HDL-associated apoprotein A-I and cholesterol esters by 16 tissues in the rat in vivo and by adrenal cells and hepatocytes in vitro. J. Biol. Chem. 260:744-750.

10. Whitlock, M. E., T. L. Swenson, R. Ramakrishnan, M. T. Leonard, Y. L. Marcel, R. W. Milne, and A. R. Tall. 1989. Monoclonal antibody inhibition of cholesteryl ester transfer protein activity in the rabbit effects on lipoprotein composition and high density lipoprotein cholesteryl ester metabolism. J. Clin. Invest. 84:129-137.

11. Brown, M. L., A. Inazu, C. B. Hesler, L. B. Agellon, C. Mann, M. E. Whitlock, Y. L. Marcel, R. W. Milne, J. Koizumi, H. Mabuchi, R. Takeda, and A. R. Tall. 1989. Molecular basis of lipid transfer protein deficiency in a family with increased high density lipoproteins. Nature (Lond.). 342:448-451.

12. Walsh, A., Y. Ito, and J. L. Breslow. 1988. High levels of human apolipoprotein A-I in transgenic mice result in increased plasma levels of small high density lipoprotein (HDL) particles comparable to human $\mathrm{HDL}_{3}$. J. Biol. Chem. 264:6488-6494. 
13. Chajek-Shaul, T., T. Hayek, A. Walsh, and J. L. Breslow. 1991. Human apo A-I expression in transgenic mice alters HDL particle size distribution and diminishes selective uptake of HDL cholesteryl esters. Proc. Natl. Acad. Sci. USA. 88:6731-6735.

14. Rubin, E. M., B. Y. Ishida, S. M. Clift, and R. M. Krauss. 1991. Expression of human apolipoprotein A-I in transgenic mice results in reduced plasma levels of murine apolipoprotein A-I and the appearance of two new high density lipoprotein size subclasses. Proc. Natl. Acad. Sci. USA. 88:434-438.

15. Agellon, L. B., A. Walsh, T. Hayek, P. Moulin, X. C. Jiang, S. A. Shelanski, J. L. Breslow, and A. R. Tall. 1991. Reduced high density lipoprotein cholesterol in human cholesteryl ester transfer protein transgenic mice. J. Biol. Chem. 266:10796-10801.

16. Blanche, P. J., E. L. Gong, T. M. Forte, and A. V. Nichols. 1981. Characterization of human high density lipoproteins by gradient gel electrophoresis. Biochim. Biophys. Acta. 665:408-418.

17. Verdery, R. B., D. F. Benham, H. L. Baldwin, A. P. Goldberg, and A. V. Nichols. Measurement of normative HDL subfraction cholesterol levels by Gaussian summation analysis of gradient gels. J. Lipid Res. 30:1085-1095.

18. Swenson, T. L., C. B. Hesler, M. L. Brown, E. Quinet, P. P. Trotta, M. F. Haslanger, F. C. A. Gaeta, Y. L. Marcel, R. W. Milne, and A. R. Tall. 1989. Mechanism of cholesteryl ester transfer protein inhibitor by a neutralizing monoclonal antibody and mapping of the monoclonal antibody epitope. J. Biol. Chem. 264:14318-14326.

19. Marcel, Y. L., R. McPherson, M. Hogue, H. Czarnecka, Z. Zawadski, P. K. Weech, M. E. Whitlock, A. R. Tall, and R. W. Milne. 1990. Distribution and concentration of cholesteryl ester transfer protein in plasma of normolipemic subjects. J. Clin. Invest. 85:10-17.

20. Bilheimer, D. W., S. Eisenberg, and R. L. Levy. 1972. The metabolism of very low density lipoprotein proteins. Preliminary in vitro and in vivo observations. Biochim. Biophys. Acta. 260:212-221.

21. McFarlane, A. S. 1958. Efficient trace-labelling of proteins with Iodine. Nature (Lond.). 182:53.

22. Stein, Y., Y. Dabah, G. Hollander, G. Halperin, and O. Stein. 1983. Metabolism of HDL-Cholesteryl ester in the rat studied with a nonhydrolyzable analog cholesteryl linoleyl ether. Biochim. Biophys. Acta. 752:98-105.

23. Matthews, C. M. 1957. The theory of tracer experiments with ${ }^{131}$ I-labelled plasma proteins. Phys. Med. Biol. 2:36-53.

24. Barter, P. J., and M. E. Jones. 1980. Kinetic studies of the transfer of esterified cholesterol between human plasma low and high density lipoproteins. J. Lipid Res. 21:238-249.

25. Ha, Y. C., L. B. F. Chang, and P. J. Barter. 1985. Effects of injecting exogenous lipid transfer protein into rats. Biochim. Biophys. Acta. 833:203-210.

26. Yamashita, S., D. L. Sprecher, N. Sakai, Y. Matsuzuawa, S. Tarui, and D. Y. Hui. 1990. Accumulation of apolipoprotein E-rich high density lipoproteins in hyperalphalipoproteinemic human subjects with plasma cholesteryl ester transfer protein deficiency. J. Clin. Invest. 86:688-695.

27. Inazu, A., M. L. Brown, C. B. Hesler, L. B. Agellon, J. Koizumi, K. Takata, Y. Maruhama, H. Mabuchi, and A. R. Tall. 1990. Increased high density lipoprotein levels caused by a common cholesteryl-ester transfer protein gene mutation. N. Engl. J. Med. 323:1234-1238. 